\title{
RESONANCE EXPANSIONS AND RAYLEIGH WAVES
}

\author{
Plamen Stefanov
}

\section{Introduction}

In this paper we study expansions of solutions of the wave equation in a compact set with initial data supported in the same set. We consider the general framework of the "black box scattering" introduced by Sjöstrand and Zworski [SjZ] (see sec. 2). In particular, this includes the classical case of scattering by obstacle with Dirichlet or Neumann boundary conditions and metric perturbations of the Laplacian with a metric equal to the Euclidean one outside a large ball. Denote by $\mathcal{U}(t)$ the solution group corresponding to the wave equation in the energy space and let $\chi$ be the multiplication with a compactly supported function $\chi(x)$ equal to 1 on some compact set containing the "black box" (the scatterer). Then we are interested in asymptotic expansions of $\chi \mathcal{U}(t) \chi$, as $t \rightarrow \infty$.

If we study the wave equation in a bounded domain, then one can use the Fourier method to get expansion of $\mathcal{U}(t)$ in terms of the eigenvalues and eigenfunctions of the corresponding Laplacian (with self-adjoint boundary conditions). In the case under consideration, one gets expansions in terms of the resonances and resonance states. This has been confirmed in the non-trapping case by LaxPhillips [LP] and Vainberg [Va1] in odd dimensions (see also [Va2]) and in the black box setting by Tang and Zworski [TZ2]. In this case,

$$
\chi U(t) \chi g=\sum_{\operatorname{Im} \lambda_{j} \leq A} \sum_{m=1}^{m_{j}} e^{i t \lambda_{j}} t^{m-1} w_{j, m}(x)+O\left(e^{-(A-\varepsilon) t}\right) g, \quad g \in \mathcal{D}, \varepsilon>0 .
$$

Here $u(t, x)=U(t) g$ is the solution to the wave equation with initial data $\left.u\right|_{t=0}=$ $0,\left.u_{t}\right|_{t=0}=g$ (see also sec. 2), $\mathcal{D}$ is the domain of the corresponding Hamiltonian and $m_{j}$ is the order of singularity of the Laurent expansion of the resolvent at the resonance $\lambda=\lambda_{j}$. The functions $w_{j, m}(x)$ are resonance states (see also section 3). In this paper we accept the convention that resonances lie in the upper half-plane $\operatorname{Im} \lambda>0$. Note that the sum above is finite.

Much less is known in case of trapping scatterers. We will study here systems with a sequence of resonances $\lambda_{j}$ converging rapidly to the real axis, i.e., $\operatorname{Im} \lambda_{j}=$ $O\left(\left|\lambda_{j}\right|^{-\infty}\right)$. Such "almost real" resonances exist for example in the classical obstacle scattering assuming the existence of non-degenerate elliptic periodic ray [SV2] (see also [TZ1], [S]); for the system of linear elasticity in exterior domain

Received September 5, 2000.

Supported by NSF Grant DMS-0070823. 
with Neumann boundary conditions [SV1], [SV2], [Vo], [S2]; for transparent obstacles [PV]. Recently, Tang and Zworski [TZ2] obtained for the first time an expansion of the type (1) for trapping systems (having "almost real" resonances) in the black box setting. They showed that

$$
\chi U(t) \chi g=\sum_{\operatorname{Im} \lambda_{j} \leq\langle\lambda\rangle-K-1} \sum_{m=1}^{m_{j}} e^{i t \lambda_{j}} t^{m-1} w_{j, m}(x)+E_{K}(t) g, \quad g \in \mathcal{D}^{M},
$$

where $K \gg 1, M \gg 1$. For the error term we have $\left\|E_{K}\right\|_{\mathcal{D}^{M} \rightarrow \mathcal{H}} \leq C_{N} t^{-N}$, if the space dimension $n$ is odd, $\left\|E_{K}\right\|_{\mathcal{D}^{M} \rightarrow \mathcal{H}} \leq t^{-n+1}$ for $n$ even and $N$ can be chosen arbitrary large by choosing $M$ large enough. This expansion is proved under the following separation condition: for $K \gg 1, \exists k>0$, such that $|\lambda-\mu| \geq$ $C \max \{|\lambda|,|\mu|\}^{-k}$ for any two distinct resonances $\lambda$ and $\mu$ in $\operatorname{Im} \lambda \leq\langle\lambda\rangle^{-K}$ and the algebraic multiplicities of those resonances are uniformly bounded. The sum above is infinite and the outer sum is absolutely convergent, while the absolute convergence of the double sum is unclear. The main argument in proving (2) is showing that the cut-off resolvent is polynomially bounded on a contour around each resonance near the real axis. This estimate relies on a priori exponential estimate of the resolvent first observed by Zworski [Z] and on the "semi-classical maximum principle" [TZ1, Lemma 2] (see also Lemma 1 below), which in turn is a significant improvement over [SV2, Lemma 1]. The approach in [TZ2] is used in [CZ] to obtain resonance expansions in two hyperbolic cases where the separation condition holds. In a recent paper, Burq and Zworski [BZ] showed that one can sum up in (2) over resonances $\lambda_{j}$ with $\left|\lambda_{j}\right| \leq t^{\varepsilon}$ with $\varepsilon=\varepsilon(M, K)>0$ and this gives an error term of the same type with larger $K$ and $M$ but without any assumptions on the resonances.

In this paper we present a generalization of the result in [TZ2]. We study a case, where the resonances near the real axis are separated from the other resonances by a polynomial region of the type $\langle\lambda\rangle^{-K} \leq \operatorname{Im} \lambda \leq\langle\lambda\rangle^{-K+2 n^{\#}+\varepsilon}$, $\varepsilon>0, K \gg 1$ and our main result is formulated in Theorem 1. We do not assume however that the resonances below that region are separated from each other. Our assumption is satisfied for example for the system of linear elasticity with Neumann boundary conditions and we discuss this in section 4 . The general case remains open. It should be noted also that our results can be also formulated in the semiclassical setting.

Below we will denote by $C$ different positive constants that may change from line to line.

\section{Assumptions and main result}

We will recall briefly the black box scattering formalism as introduced in [SjZ] (see also [TZ2]). Let $\mathcal{H}$ be a complex Hilbert space with orthogonal decomposition

$$
\mathcal{H}=\mathcal{H}_{R_{0}} \oplus L^{2}\left(\mathbf{R}^{n} \backslash B_{R_{0}}\right)
$$


where $B_{R_{0}}:=\left\{x \in \mathbf{R}^{n} ;|x|<R_{0}\right\}$ and $R_{0}$ is fixed. Let $P$ be a self-adjoint operator in $\mathcal{H}$ with domain $\mathcal{D} \subset \mathcal{H}$ such that $\mathbf{1}_{\mathbf{R}^{n} \backslash B_{R_{0}}} \mathcal{D}=H^{2}\left(\mathbf{R}^{n} \backslash B_{R_{0}}\right)$, $\mathbf{1}_{\mathbf{R}^{n} \backslash B_{R_{0}}} P=-\left.\Delta\right|_{\mathbf{R}^{n} \backslash B_{R_{0}}},(P+i)^{-1}$ is compact and $P \geq 0$. For simplicity, we will assume that $P$ has no eigenvalues. Those conditions guarantee that $R(\lambda):=\left(P-\lambda^{2}\right)^{-1}: \mathcal{H} \rightarrow \mathcal{D}$ admits a meromorphic extension as an operator from $\mathcal{H}_{\text {comp }}$ to $\mathcal{D}_{\text {loc }}$ from the lower half-plane to the whole complex plane when $n$ is odd, and to the logarithmic plane if $n$ is even. The poles of this extension that we will still denote by $R(\lambda)$ are called resonances and we will denote the set of resonances by $\mathcal{R}(P)$. Each resonance has finite multiplicity defined as the rank of the residue and the Laurant expansion at the pole has finite order of singularity not exceeding the multiplicity. We will always include resonances according to their multiplicities. We also assume that for the "reference operator" $P$ \#, constructed from $P$, on $\mathcal{H}_{R_{0}} \oplus L^{2}\left(M \backslash B_{R_{0}}\right), M:=(\mathbf{R} \backslash R \mathbf{Z})^{n}$ for some $R>R_{0}$, we have

$$
N\left(P^{\#}, r\right):=\#\left\{\lambda^{2} \in \operatorname{spec} P^{\#} ; 0 \leq \lambda \leq r\right)=O\left(r^{n^{\#}}\right)
$$

with some $n^{\#} \geq n$. Then (see $[\mathrm{Sj}]$ and the references herein), for some $\theta>0$ for the number of resonances $N_{\theta}(r)$ in $\{|\lambda| \leq r, \arg \lambda<\theta\}$ we have

$$
N_{\theta}(r) \leq C_{\theta} r^{n^{\#}}, \quad r \geq 1 .
$$

The solution to the wave equation $\left(\partial_{t}^{2}+P\right) u=0$ with initial conditions $\left.u\right|_{t=0}=$ $f_{1},\left.\partial_{t} u\right|_{t=0}=f_{2}$ can be expressed in the corresponding energy space by the unitary group

$$
\mathcal{U}(t)=\exp \left(\begin{array}{cc}
0 & I \\
-P & 0
\end{array}\right) t=\left(\begin{array}{cc}
\partial_{t} U(t) & U(t) \\
\partial_{t}^{2} U(t) & \partial_{t} U(t)
\end{array}\right),
$$

where $U(t): \mathcal{D}^{k} \rightarrow \mathcal{D}^{k+1}$ and $u=U(t) f$ solves the wave equation $\left(\partial_{t}^{2}+P\right) u=0$ with initial data $\left.\left(u, u_{t}\right)\right|_{t=0}=(0, f)$. In particular, this shows that in order to study the local behavior of $\mathcal{U}(t)$, it is enough to study $U(t)$. We will also use the notation $\mathcal{D}^{k}:=(P+i)^{-k} \mathcal{H}$.

We are ready now to formulate our main result.

Theorem 1. Assume that for some $K>7 n^{\#} / 2$ there are no resonances of $P$ in $\langle\lambda\rangle^{-K} \leq \operatorname{Im} \lambda \leq\langle\lambda\rangle^{-K+2 n^{\#}+\varepsilon}, \varepsilon>0$, for $|\lambda| \gg 1$. Then

(4) $\chi U(t) \chi g=$

$$
-i \sum_{l=1}^{\infty} \sum_{\substack{\lambda_{j} \in \mathcal{R}(P) ; \operatorname{Re} \lambda_{j} \in I_{l} \\ \operatorname{Im} \lambda_{j}<\langle\lambda\rangle-K}} \chi \operatorname{Res}\left\{e^{i t \lambda} R(\lambda), \lambda_{j}\right\} \chi g+E_{K}(t) g, \quad g \in \mathcal{D}^{M},
$$

$M \geq(K+1) / 2$, where $\operatorname{Res}\left\{f(z), z_{0}\right\}$ stands for the residue of $f$ at the pole $z_{0}$. Here $I_{l}=\left[a_{l}, b_{l}\right], a_{l}<b_{l}<a_{l+1}$ is any sequence of intervals such that $\operatorname{dist}\left\{I_{l}, I_{l+1}\right\}=b_{l+1}-a_{l} \geq a_{l}^{-k}, k>n^{\#}$, and $\operatorname{Re}\left(\mathcal{R}(P) \cap\left\{\operatorname{Im} \lambda<\langle\lambda\rangle^{-K}\right\}\right) \subset$ $\cup_{l} I_{l}$. The outer sum is absolutely convergent. The error term $E_{K}(t)$ satisfies $\left\|E_{K}(t)\right\|_{\mathcal{D}^{M} \rightarrow \mathcal{H}} \leq C_{N} t^{-N}, N=(2 M-K) /\left(K-n^{\#}+1+\varepsilon\right), n$ odd, and $\left\|E_{K}(t)\right\|_{\mathcal{D}^{M} \rightarrow \mathcal{H}} \leq C t^{-\min (n-1, N)}, n$ even 
Remark 1. Even though the outer sum is absolutely convergent, we cannot guarantee that the double sum is absolutely convergent or even convergent (see also [TZ2]).

Remark 2. For any resonance $\lambda_{j}$, we have

$$
\chi \operatorname{Res}\left\{e^{i t \lambda} R(\lambda), \lambda_{j}\right\} \chi g=\sum_{m=0}^{m_{j}-1} e^{i \lambda_{j} t} t^{m} w_{j, q}(x)
$$

(compare with (1)). Since $\operatorname{Im} \lambda_{j}>0$, each term above decreases exponentially fast, while the error term $E_{k}(t)$ tends to zero only at a polynomial rate! The exponential rate of decay however depends on $\lambda$ and becomes small for large $\lambda$ 's. We do not have effective lower bounds on $w_{j, q}$ 's, but based on the upper bounds (see (20)), we expect that the terms in (4) corresponding to resonances close to the upper boundary $\operatorname{Im} \lambda=\langle\lambda\rangle^{-K}$ would be comparable to the error term $E_{K}(t)$, while if $\lambda_{j}$ is close to the real axis, for example exponentially close, then the corresponding terms will dominate over $E_{K}(t)$. Also, we may have the accumulative effect of infinite many $w_{j, q}$.

Remark 3. The assumption of a resonance free zone $\langle\lambda\rangle^{-K} \leq \operatorname{Im} \lambda \leq$ $\langle\lambda\rangle^{-K+2 n^{\#}+\varepsilon},|\lambda| \gg 1$ can be relaxed. It is enough to assume that in this zone we have the following property: There exist $k_{0}>0$ and $n_{0}>0$ such that for any $a>0$ large enough the number of resonances in this zone with $a \leq \operatorname{Re} \lambda \leq a+a^{-k_{0}}$ does not exceed $n_{0}$. Then Theorem 1 still holds with different $M$ and $N$ depending on $k_{0}, n_{0}$. Also, we may have to deform the contour $\operatorname{Im} \lambda=\langle\lambda\rangle^{-K}$ in order to include in (4) a possible sequence of resonances above it that may converge to this curve faster than any polynomial of $1 /|\lambda|$ as in [BZ]. Notice that the so relaxed assumption is satisfied if the resonances there are "separated" as in [TZ2].

\section{Proof of Theorem 1}

Technically, it is convenient to work in the semiclassical setting. Set $P(h):=$ $h^{2} P$, where $0<h<1$. The poles of the analytic continuation of the resolvent $R(z, h):=(P(h)-z)^{-1}: \mathcal{H}_{\text {comp }} \rightarrow \mathcal{H}_{\text {loc }}$ from $\Omega \cap\{\operatorname{Im} z<0\}$ to $\Omega$, where $\Omega$ is a neighborhood of some energy level $E>0$ are resonances of $P(h)$ and with some abuse of notation we will denote them by $\mathcal{R}(P(h))$. Thus the relationship between the resonances $\lambda \in \mathcal{R}(P)$ of $P$ and the resonances $z \in \mathcal{R}(P(h))$ is given by $\lambda=h^{-1} z^{1 / 2}$. Here and below we denote by $\chi$ the multiplication by a compactly supported function $\chi(x)$ equal 1 on $B_{R}, R>R_{0}$. More precisely, $\chi=\mathbf{1}_{\mathcal{H}_{R_{0}}} \oplus \tilde{\chi}$, where $\tilde{\chi}$ is the multiplication by the restriction $\tilde{\chi}(x)$ of $\chi(x)$ on $\mathbf{R}^{n} \backslash B_{R_{0}}$. It is convenient to assume that $0 \leq \chi \leq 1$. We will use the notation $R_{\chi}(z, h):=\chi R(z, h) \chi$ and $R_{\chi}(\lambda):=\chi R(\lambda) \chi$. Sometimes we will denote $R_{\chi}(z, h)$ simply by $R_{\chi}(z)$.

First we formulate an a priori exponential estimate on the resolvent. As mentioned in the Introduction, this estimate was first observed by M. Zworski $[\mathrm{Z}]$. In this generality it was proved in [TZ1], [TZ2]. 
Proposition 1. Under the conditions above, let $\Omega$ be a simply connected compact neighborhood of $E>0$ in $\operatorname{Re} z>0$. Then for $0<h<h_{0}$ with some $h_{0}>0$ we have

$$
\begin{aligned}
& \left\|R_{\chi}(z, h)\right\|_{\mathcal{H} \rightarrow \mathcal{H}} \leq e^{C_{\Omega} h^{-n \#} \ln (1 / g(h))}, \quad \text { for } z \in \Omega, \\
& \qquad\left|z-z_{j}\right| \geq g(h), \quad \forall z_{j} \in \mathcal{R}(P(h)), \quad g(h) \ll 1 .
\end{aligned}
$$

The following "semi-classical maximum principle" is a modification of [TZ2, Lemma 4.1] (see also [TZ1, Lemma 2] and [SV2, Lemma 1]). We formulate here a more general version of this lemma suitable for our purposes.

Lemma 1. Fix $k>0, n^{\#}>0$. Let $0<h<1$ and $a(h) \leq b(h)$. Suppose that $F(z, h)$ is a holomorphic function of $z$ defined in a neighborhood of

$$
\Omega(h)=[a(h)-5 w(h), b(h)+5 w(h)]+i\left[-S_{-}(h), S_{+}(h) h^{-n^{\#}-\varepsilon}\right],
$$

where $0<S_{-}(h) \leq S_{+}(h) \leq w(h) h^{3 n^{\#} / 2+2 \varepsilon}$, $\varepsilon>0$ and $w(h) \rightarrow 0$, as $h \rightarrow 0$. If $F(z, h)$ satisfies

$$
\begin{aligned}
& |F(z, h)| \leq A e^{A h^{-n \#} \ln (1 / h) \quad \text { on } \Omega(h),} \\
& |F(z, h)| \leq M(h) \quad \text { on }[a(h)-5 w(h), b(h)+5 w(h)]-i S_{-}(h)
\end{aligned}
$$

with $M(h) \rightarrow \infty$, as $h \rightarrow 0$, then there exists $h_{1}=h_{1}\left(S_{-}, S_{+}, A, k, \varepsilon\right)>0$ such that

$$
\begin{aligned}
|F(z, h)| & \leq 2 e^{3} M(h), \\
\forall z & \in \tilde{\Omega}:=[a(h)-w(h), b(h)+w(h)]+i\left[-S_{-}(h), S_{+}(h)\right], \text { for } h \leq h_{1} .
\end{aligned}
$$

Sketch of the Proof. The proof follows those of [TZ2, Lemma 4.1] and [TZ1, Lemma 2] with some modifications. Set

$$
f(z, h):=\left(\pi \alpha^{2}\right)^{-1 / 2} \int_{a(h)-3 w(h)}^{b(h)+3 w(h)} \exp \left(-\frac{(x-z)^{2}}{\alpha^{2}}\right) d x, \quad \alpha:=S_{+}(h) h^{-n^{\#}-\varepsilon} .
$$

Then $f(z, h)$ is holomorphic in $\Omega(h)$ and for $h \ll 1$ satisfies:

$$
\begin{array}{ll}
|f(z, h)| \leq e \quad \text { in } \Omega(h), & \\
|f(z, h)| \geq \frac{1}{2} \quad \text { in } \tilde{\Omega}(h), & \\
|f(z, h)| \leq C e^{-h^{-n}-\varepsilon}, & \text { in } \Omega(h) \cap\{\operatorname{Re} z \leq a(h)-4 w(h) \\
& \text { or } \operatorname{Re} z \geq b(h)+4 w(h)\} .
\end{array}
$$

Next, we apply the maximum principle to the function

$$
G(z, h):=e^{i z / S_{+}(h)} f(z, h) F(z, h)
$$

in $\Omega(h)$. On the upper part of $\partial \Omega(h)$, the exponential function above compensates for the exponential growth (5) of $F$, so $|G|=o(1)$, as $h \rightarrow 0$, there. On the sides, the exponential function is bounded by $\exp \left(S_{-}(h) / S_{+}(h)\right)<e$ and the exponential growth of $F$ is controlled by $f$ in view of $(9)$ so we have 
again $|G|=o(1)$. On the lower part $\operatorname{Im} z=-S_{-}(h)$ of $\partial \Omega(h)$, we have $|G| \leq e|f F| \leq e^{2} M(h)$ by (7) and (6). Thus $|G| \leq e^{2} M(h)$ on $\partial \Omega(h)$ for $h \ll 1$. By the maximum principle, this is true in $\Omega(h)$ as well. Using the fact that $\left|e^{i z / S_{+}(h)}\right| \geq 1 / e$ on $\partial \tilde{\Omega}(h)$ and $(8)$, we get

$$
\frac{1}{2 e}|F| \leq\left|e^{i z / S_{+}(h)}\right||F||f| \leq e^{2} M(h),
$$

which proves the lemma.

Typically, Lemma 1 is applied to $R_{\chi}(z)$ (or to some multiple of it) and then one can use the classical estimate $\left\|R_{\chi}(z)\right\| \leq 1 /|\operatorname{Im} z|$ in the lower half-plane $\operatorname{Im} z<0$, so in this case $M(h)=1 / S_{-}(h)$.

Let $\Omega(h)$ be as above with

$$
w(h):=h^{k}, \quad S_{+}(h):=h^{k+3 n^{\#} / 2+2 \varepsilon}, \quad k>0, \varepsilon>0 .
$$

Let $z_{1}(h), z_{2}(h) \ldots z_{p}(h)$ be all resonances in $\Omega(h)$ and denote by $m_{1}, \ldots, m_{p}$ the corresponding multiplicities. Set $m:=m_{1}+\cdots+m_{p}$. Assume also that all resonances in $\Omega(h)$ actually lie in $[a(h), b(h)]+i\left[0, S_{-}(h)\right]$ with $S_{-}(h):=$ $h^{k+5 n^{\#} / 2+2 \varepsilon}$. Let

$$
\tilde{z}_{j}(h):=\bar{z}_{j}(h)-2 i S_{-}(h), \quad j=1 \ldots p,
$$

where the bar denotes complex conjugate (see Figure 1). Note that $z_{j}$ and $\tilde{z}_{j}$ are symmetric about the line $\operatorname{Im} z=-S_{-}(h)$ (the lower part of $\partial \Omega(h)$ ). Set

$$
G(z, h):=\frac{\left(z-z_{1}\right)^{m_{1}} \ldots\left(z-z_{p}\right)^{m_{p}}}{\left(z-\tilde{z}_{1}\right)^{m_{1}} \ldots\left(z-\tilde{z}_{p}\right)^{m_{p}}} .
$$

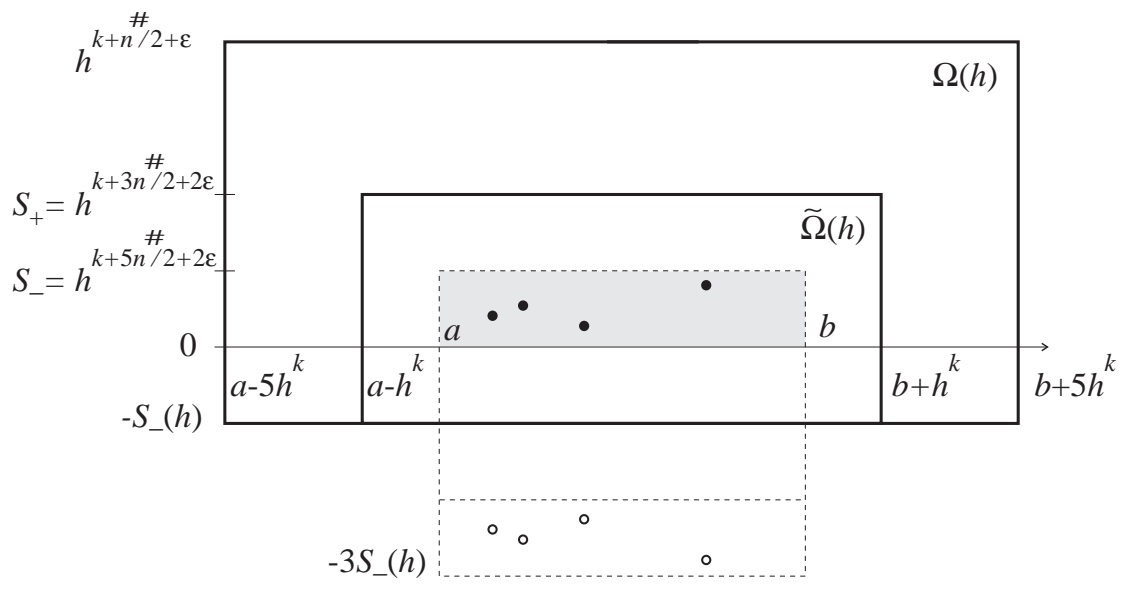

Figure 1: Resonances $z_{j}$ are denoted by $\bullet$; $\tilde{z}_{j}$ are denoted by $\circ$ 
It is easy to see that

$$
|G(z, h)| \leq 1 \quad \text { for } \operatorname{Im} z \geq-S_{-}(h) .
$$

The function $F:=G R_{\chi}$ is holomorphic in $\Omega(h)$ and satisfies the assumptions of Lemma 1 if we assume that $\operatorname{dist}(\partial \Omega(h), \mathcal{R}(P)) \geq C h^{K}$ with some $K>0$. Indeed, by Proposition 1, the exponential estimate is satisfied in the complement (in $\Omega(h)$ ) of disks centered at the resonances with radii $h^{N}$ with fixed $N \gg 1$ (see [TZ2]). Those disks may intersect but can form connected unions of size not more than $O\left(h^{N-n^{\#}}\right)$ that will stay away from $\partial \Omega(h)$. Since $F$ is holomorphic in those disks, applying the maximum principle, we get the exponential estimate in the whole $\Omega(h)$ (see also the proof of Theorem 1 in $[\mathrm{S}]$ ). Note that this condition and therefore the exponential estimate are automatically satisfied if we increase $\varepsilon$ and $k$. On the lower part of $\partial \Omega(h)$ we have the resolvent estimate $\left\|R_{\chi}(z)\right\| \leq 1 /|\operatorname{Im} z|$ for $\operatorname{Im} z<0$ and (10), thus $\left\|G R_{\chi}\right\| \leq 1 / S_{-}(h)$ on $\partial \Omega(h) \cap\left\{\operatorname{Im} z=-S_{-}(h)\right\}$. By Lemma $1,\left\|G R_{\chi}\right\| \leq 2 e^{3} / S_{-}(h)$ in $\tilde{\Omega}(h)$ for $h$ small enough.

We now claim that

$$
1 / C \leq|G(z, h)| \quad \text { on } \partial \tilde{\Omega}(h) .
$$

with some $C>0$ depending only on the constant in (3). It is enough to estimate $\left(z-\tilde{z}_{j}\right) /\left(z-z_{j}\right)$ on $\partial \tilde{\Omega}(h)$. We have

$$
\begin{aligned}
&\left|\frac{z-\tilde{z}_{j}}{z-z_{j}}-1\right|=\left|\frac{z_{j}-\tilde{z}_{j}}{z-z_{j}}\right| \leq \frac{4 S_{-}(h)}{h^{k+3 n^{\#} / 2+2 \varepsilon} / 2}=8 h^{n^{\#}} \\
& \forall z \in \partial \tilde{\Omega}(h) \backslash\left\{\operatorname{Im} z=-S_{-}(h)\right\}
\end{aligned}
$$

for $0<h<1 / 2$ because $\left|z-z_{j}\right| \geq h^{k+3 n^{\#} / 2+2 \varepsilon}-h^{k+5 n^{\#} / 2+2 \varepsilon} \geq h^{k+3 n^{\#} / 2+2 \varepsilon} / 2$ for $h<1 / 2$ if $\operatorname{Im} z=h^{k+3 n^{\#} / 2+2 \varepsilon}$ and we have greater lower bound for $z$ on the right and left sides of $\partial \tilde{\Omega}(h)$. Therefore,

$$
\left|\frac{z-\tilde{z}_{j}}{z-z_{j}}\right|^{m_{j}} \leq\left(1+8 h^{n^{\#}}\right)^{m_{j}}, \quad \forall z \in \partial \tilde{\Omega}(h) \backslash\left\{\operatorname{Im} z=-S_{-}(h)\right\}
$$

On the other hand, (12) is trivially true on the lower side $\operatorname{Im} z=-S_{-}(h)$ of $\partial \tilde{\Omega}(h)$ because $\left|\left(z-\tilde{z}_{j}\right) /\left(z-z_{j}\right)\right|=1$ there. Since $(1+x)^{1 / x}<e, 0<x<\infty$, we get

$$
|G(z, h)| \leq\left(1+8 h^{n^{\#}}\right)^{m_{1}+\cdots+m_{p}}=\left(1+8 h^{n^{\#}}\right)^{m} \leq\left(1+8 h^{n^{\#}}\right)^{C h^{-n^{\#}}} \leq e^{8 C} .
$$

This proves our claim.

The estimate we got on $G R$ and (11) together imply $\left\|R_{\chi}\right\|=O\left(1 / S_{-}(h)\right)=$ $O\left(h^{-k-5 n^{\#} / 2-2 \varepsilon}\right)$ on $\partial \tilde{\Omega}(h)$. We have therefore proved the following.

Lemma 2. Assume that all resonances in

$$
\left[a(h)-6 h^{k}, b(h)+6 h^{k}\right]+i\left[0, h^{k+n^{\#} / 2}\right]
$$

lie in $[a(h), b(h)]+i\left[0, h^{k+5 n^{\#} / 2+\varepsilon}\right], \varepsilon>0$. Then

$$
\left\|R_{\chi}\right\|=O\left(h^{-k-5 n^{\#} / 2-\varepsilon}\right) \quad \text { on } \partial \tilde{\Omega}(h),
$$


where $\tilde{\Omega}(h):=\left[a(h)-h^{k}, b(h)+h^{k}\right]+i\left[-h^{k+5 n^{\#} / 2+\varepsilon}, h^{k+3 n^{\#} / 2+\varepsilon}\right]$.

We note that we increased $\Omega(h)$ in order to make sure that all resonances outside the original $\Omega(h)$ are at distance at least $h^{K}$ with some $K>0$ and we also replaced $2 \varepsilon$ by $\varepsilon$.

The rest of the proof follows closely that of [TZ2]. We have

$$
\chi U(t) \chi g=\frac{1}{2 \pi} \int_{-\infty-i \alpha}^{\infty-i \alpha} e^{i t \lambda} R_{\chi}(\lambda) g d \lambda, \quad g \in \mathcal{D}, \alpha>0 .
$$

In what follows we will assume that $g$ is compactly supported (we can always assume that). Assume first that $n$ is odd. Then we are going to lift the contour of integration to the pole-free zone such that $R_{\chi}$ is polynomially bounded on the new contour as well. Using (3) one can show (see $[\mathrm{S}],[\mathrm{SjV}]$ ) that for any $k>n^{\#}+1$ all resonances in $\Lambda:=\left\{\operatorname{Im} \lambda\left\langle\langle\lambda\rangle^{-K}\right\}\right.$ can be grouped into clusters $U_{l}$ with $\operatorname{Re}\left(U_{l}\right) \subset I_{l}$, where the intervals $I_{l}:=\left(a_{l}, b_{l}\right)$ are as in Theorem 1 with the properties: $\left|I_{l}\right|=O\left(\lambda^{-k+1+n^{\#}}\right)$ and $\operatorname{dist}\left(I_{l}, I_{l+1}\right) \geq 4 \lambda^{-k+1}, 1 \ll \lambda \in I_{l}$. Set $h=h_{l}:=a_{l}^{-1}$ and $P(h):=h^{2} P, h \in\left\{h_{l}\right\}_{l=1}^{\infty}$. Then under the scaling $\lambda \mapsto h^{2} \lambda^{2}=: z$ the interval $I_{l}$ transforms into $\left(1, b_{l}^{2} / a_{l}^{2}\right)=:(a(h), b(h))$ and we get that there are no resonances $z$ of $P(h)$ (such that $\lambda(z) \in \Lambda$ ) with real parts in $\left[a(h)-7 h^{k}, b(h)+7 h^{k}\right] \backslash[a(h), b(h)]$. Simple calculations show that the condition $\operatorname{Im} \lambda=\langle\lambda\rangle^{-K}$ implies $\operatorname{Im} z=(2+o(1)) h^{K+1}$. Therefore the

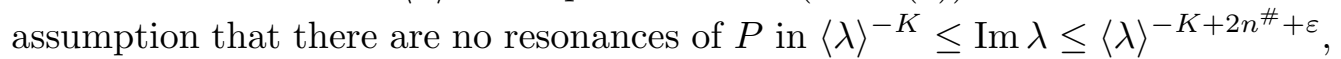
$\lambda \gg 1$, guarantees that all resonances of $P(h)$ in $\left[a(h)-7 h^{k}, b(h)+7 h^{k}\right]+$ $i\left[0, h^{K-2 n^{\#}+1-\varepsilon}\right]$ actually lie in $[a(h), b(h)]+i\left[0,3 h^{K+1}\right]$ for $h$ small enough. So in particular they lie in $[a(h), b(h)]+i\left[0, h^{K+1-\varepsilon / 2}\right]$ for $h$ small enough. Set

$$
k:=K-5 n^{\# / 2}+1-\varepsilon \text {. }
$$

Then $k>n^{\#}+1$ for $\varepsilon \ll 1$ and we are in position to apply Lemma 2 with $\varepsilon$ replaced by $\varepsilon / 2$ to get $\left\|R_{\chi}(z)\right\|=O\left(h^{-K-1+\varepsilon / 2}\right)$ on $\partial \tilde{\Omega}(h)$. We also used the classical estimate $\left\|R_{\chi}(z)\right\| \leq 1 /|\operatorname{Im} z|$ for $\operatorname{Im} z<0$, so $M(h)=1 / S_{-}(h)$ in $(6)$.

Applying the transform $z \mapsto h^{-1} \sqrt{z}=: \lambda, \lambda \in\left[a_{l}, b_{l}\right], h=1 / a_{l}$, we get that

$$
\left\|R_{\chi}(\lambda)\right\|=O\left(|\lambda|^{K-1}\right) \quad \text { on } \Gamma_{l},
$$

where $\Gamma_{l}$ is asymptotically close to the boundary of the rectangle

$$
\begin{aligned}
& a_{l}-\frac{1}{2} a_{l}^{-k+1} \leq \operatorname{Re} \lambda \leq b_{l}+\frac{1}{2} a_{l}^{-k+1}, \\
& -\frac{1}{2} a_{l}^{-k-5 n^{\#} / 2-\varepsilon / 2+1} \leq \operatorname{Im} \lambda \leq \frac{1}{2} a_{l}^{-k-5 n^{\#} / 2-\varepsilon / 2+1} .
\end{aligned}
$$

Since we have the freedom to perturb $a_{l}$ by $c a_{l}^{-k+1}, c \ll 1$, we can actually assume that $\Gamma_{l}$ exactly coincides with the boundary of the rectangle above.

It remains to estimate the resolvent in the gaps between the intervals $I_{l}$. We know that there are no resonances $\lambda_{j}$ in $\Lambda$ with real parts in $\left(b_{l}, a_{l+1}\right)$, $l=1,2, \ldots$ and moreover $a_{l+1}-b_{l} \geq 4 b_{l}^{-k+1}$. We can replace the constant 4 there by any other by increasing $k$ (this is not necessary in fact, since the 
terms $\pm 5 h^{k}$ in Lemma 1 can be replaced by $\left.\pm(1+\varepsilon) h^{k}, \forall \varepsilon>0\right)$. So, we have $a_{l+1}-b_{l} \geq 20 b_{l}^{-k}$. Assume first that $a_{l+1}-b_{l} \leq b_{l}^{-1}$. Set $h:=b_{l}^{-1}$, apply Lemma 1 and then go back to the $\lambda$ variables. We get that

$$
\left\|R_{\chi}(\lambda)\right\|=O\left(|\lambda|^{K-n^{\#} / 2-1+\varepsilon}\right) \quad \text { in } \Pi_{l},
$$

where $\Pi_{l}$ is given by

$$
\Pi_{l}:=\left[b_{l}+5 b_{l}^{-k}, a_{l+1}-5 b_{l}^{-k}\right]+\frac{i}{2}\left[-a_{l}^{-k-3 n^{\#} / 2-2 \varepsilon}, a_{l}^{-k-3 n^{\#} / 2-2 \varepsilon}\right] .
$$

Clearly the curve $\gamma:=\operatorname{Im} \lambda=\frac{1}{2}\langle\lambda\rangle^{-K+n^{\#}-1-\varepsilon}$ (see (14)) lies below the upper boundary of $\Pi_{l}$, so we have in particular that (17) is satisfied on $\gamma \cap \Pi_{l}$. Note that $\Pi_{l}$ and the rectangle (16) enclosed by $\Gamma_{l}$ overlap for $l$ large enough. Similarly, $\Pi_{l}$ and $\Gamma_{l+1}$ intersect for the same reason. If the assumption $a_{l+1}-b_{l} \leq b_{l}^{-1}$ is not satisfied, we can cover $\left(b_{l}, a_{l+1}\right)$ by a sequence of overlapping intervals of length $O\left(|\lambda|^{-1}\right)$ and apply similar arguments to get that the polynomial estimate (17) holds on $\gamma \cap\left\{b_{l}+5 b_{l}^{-k} \leq \operatorname{Re} \lambda \leq a_{l+1}-6 a_{l+1}^{-k}\right\}$.

We are ready to construct the contour $\Gamma$. For $b_{l}+5 b_{l}^{-k}<\operatorname{Re} \lambda<a_{l+1}-7 a_{l+1}^{-k}$, we choose $\Gamma$ to coincide with $\gamma$. For $a_{l} \leq \lambda \leq b_{l}$, we set $\Gamma$ to be that part of $\Gamma_{l}$ that lies above $\gamma$ (see Figure 2). We define $\Gamma$ in $\operatorname{Re} \lambda \leq 0$ as a symmetric image of $\Gamma$ in $\operatorname{Re} \lambda \geq 0$.

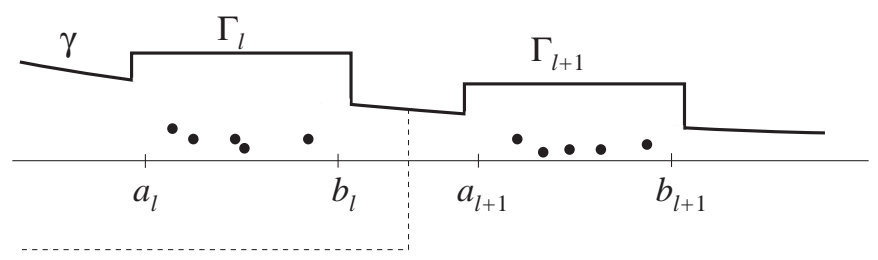

Figure 2: The contour $\Gamma$

To finish the proof of Theorem 1, we will lift the contour of integration in (13). To this end, let us choose the following closed (positively oriented) curve $\mathcal{C}_{l}$ : the upper part is $\Gamma \cap\left\{|\operatorname{Re} \lambda| \leq \frac{1}{2}\left(b_{l}+a_{l+1}\right)\right\}$, the lower part is $\{\operatorname{Im} \lambda=$ $\left.-\alpha,|\operatorname{Re} \lambda| \leq \frac{1}{2}\left(b_{l}+a_{l+1}\right)\right\}$, and the sides are $\left\{\operatorname{Re} \lambda= \pm \frac{1}{2}\left(b_{l}+a_{l+1}\right),-\alpha \leq\right.$ $\left.\operatorname{Im} \lambda \leq \frac{1}{2}\langle\lambda\rangle^{-K+n^{\#}-1-\varepsilon}\right\}$. By (15) and (17), $\left\|R_{\chi}(\lambda)\right\|=O\left(|\lambda|^{K-1}\right)$ on $\mathcal{C}_{l}, \forall l$. We can improve this estimate by letting $R_{\chi}(\lambda)$ act on smoother functions in view of the inequality (see e.g. [TZ2])

$$
\left\|R_{\chi}(\lambda)\right\|_{\mathcal{D}^{M} \rightarrow \mathcal{H}} \leq C_{M}|\lambda|^{-2 M}\left\|R_{\chi^{\prime}}(\lambda)\right\|, \quad M>0
$$

where $\chi^{\prime} \in C_{0}^{\infty}$ is such that $\chi^{\prime}=1$ on $\operatorname{supp} \chi$. This yields

$$
\left\|R_{\chi}(\lambda)\right\|_{\mathcal{D}^{M} \rightarrow \mathcal{H}}=O\left(|\lambda|^{-2}\right) \quad \text { on } \mathcal{C}_{l}, \forall l \text {, with } M \geq(K+1) / 2 .
$$

As mentioned above, without loss of generality we can assume that $g$ is of compact support and $\chi=1$ on $\operatorname{supp} g$. Integrating over $\mathcal{C}_{l}$ and taking into account 
that integrals over the vertical sides tend to zero, as $l \rightarrow \infty$ in view of (20), we see that (13) transforms into

$$
\chi U(t) \chi g=\sum_{l=1}^{\infty} w_{l}(t, x)+E(t) g, \quad g \in \mathcal{D}^{M}
$$

where

$$
\begin{aligned}
& w_{l}(t, x):=\sum_{\substack{\lambda_{j} \in \mathcal{R}(P) ; \operatorname{Re} \lambda_{j} \in I_{l} \\
\operatorname{Im} \lambda_{j}<\langle\lambda\rangle-K}}-i \chi \operatorname{Res}\left\{e^{i t \lambda} R(\lambda), \lambda_{j}\right\} \chi g \\
& =\sum_{\substack{\lambda_{j} \in \mathcal{R}(P) ; \operatorname{Re} \lambda_{j} \in I_{l} \\
\operatorname{Im} \lambda_{j}<\langle\lambda\rangle-K}} e^{i \lambda_{j} t} \sum_{m=0}^{m_{j}-1} t^{m} w_{l, m}(x)
\end{aligned}
$$

and

$$
E(t) g=\frac{1}{2 \pi} \int_{\Gamma} e^{i t \lambda} R_{\chi}(\lambda) g d \lambda
$$

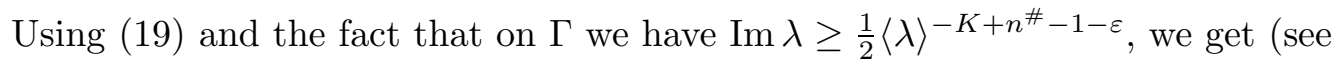
[TZ2] for more details)

$$
\begin{aligned}
\|E(t) g\|_{\mathcal{H}} & =\left\|\int_{\Gamma} e^{i t \lambda} R_{\chi}(\lambda) g d \lambda\right\| \\
& \leq C\left(\int_{1}^{\infty} e^{-t x^{-K+n}-1-\varepsilon / 2} x^{-2 M+K-1} d x+O\left(e^{-C t}\right)\right)\|g\|_{\mathcal{D}^{M}} \\
& =O\left(t^{-(2 M-K) /\left(K-n^{\#}+1+\varepsilon\right)}\right)\|g\|_{\mathcal{D}^{M} .}
\end{aligned}
$$

To finish the proof for $n$ odd, we notice that the intervals $I_{l}$ with the required properties exist because of the polynomial estimate (3) of the number of resonances. In (4) one can sum over a different family of intervals as long as $\operatorname{dist}\left\{I_{l}, I_{l+1}\right\} \geq a_{l}^{-k}, k>n^{\#}$. If $\left|I_{l}\right| \neq O\left(a_{l}^{-k+n^{\#}}\right)$, then one can split $I_{l}$ into several subintervals with gaps between them of required minimal length and then we apply what we already proved.

In the even dimensional case we have to deform the contour in a different way near $\lambda=0$ (see [TZ2]) and the contribution of $\lambda=0$ is $O\left(t^{-n+1}\right)$ (as in the unperturbed case).

The statement about the absolute convergence of the outer sum in (4) follows from the bound (15) on $\Gamma_{l}$. For $w_{l}$ (see (21)) we have

$$
\begin{aligned}
\left\|w_{l}(t, \cdot)\right\| \leq \frac{e^{t c_{l}}}{2 \pi} \oint_{\Gamma_{l}}\left\|R_{\chi}(\lambda) g\right\||d \lambda| & \\
& \leq C e^{t c_{l}} \lambda^{-2}\left|\Gamma_{l}\right|\|g\|_{\mathcal{D}^{M}} \leq C e^{t c_{l}} \lambda^{-2}\left|I_{l}\right|\|g\|_{\mathcal{D}^{M}}
\end{aligned}
$$

where $\lambda \in I_{l}$ and $c_{l}=O\left(a_{l}^{-K+1}\right)$. This estimate easily implies the convergence of the outer sum in (4) for any fixed $t$.

The estimate above grows exponentially as $t \rightarrow \infty$, which is unnatural. Below we will show that the left hand side of $(22)$ admits a similar estimate with $\exp \left(t c_{l}\right)$ 
replaced by a decaying term. Next proposition is an analogue of the classical estimate $\left\|\left(P-\lambda^{2}\right)^{-1}\right\| \leq 1 / \operatorname{dist}\left\{\lambda^{2}, \operatorname{spec}(P)\right\}$ for a self-adjoint $P$. It holds under the a priori exponential estimate (23). An estimate of this type with $q=1$ has been proved by Burq [B1], [B2] for a large class of elliptic operators in the exterior of an obstacle.

Notice that below we do not assume the resonance free zone condition of Theorem 1.

Proposition 2. Assume that $\exists q>0$, such that

$$
\operatorname{Im} \lambda_{j} \geq C_{1} e^{-C_{2}\left|\lambda_{j}\right|^{q}} \quad \text { for all resonances } \lambda_{j} .
$$

Let $d(\lambda):=\min \left\{\operatorname{dist}(\lambda, \mathcal{R}(P)),|\lambda|^{1-\delta}\right\}, \delta>0$ fixed, and set $N^{\#}:=n^{\#}+q$. Then for any $\varepsilon>0$ we have

$$
\left\|R_{\chi}(\lambda)\right\| \leq \frac{C|\lambda|^{\frac{3 N \#}{2}}-1+\varepsilon}{d(\operatorname{Re} \lambda)} \quad \text { for } 0 \leq \operatorname{Im} \lambda \leq \frac{d(\operatorname{Re} \lambda)}{21|\lambda|^{\frac{3 N \#}{2}}+\varepsilon},|\lambda| \gg 1 .
$$

Proof. Let $\lambda_{0} \gg 1$ and set $h:=1 / \lambda_{0}$. Assume that

$$
\lambda \in W\left(\lambda_{0}\right):=\left[\lambda_{0}-\frac{1}{2} d\left(\lambda_{0}\right), \lambda_{0}+\frac{1}{2} d\left(\lambda_{0}\right)\right]+i\left[0, d\left(\lambda_{0}\right) \lambda_{0}^{-N^{\#} / 2-\varepsilon}\right] .
$$

Then there are no resonances in $W\left(\lambda_{0}\right)$ for $\lambda_{0} \gg 1$. Apply the transform $z=$ $h^{2} \lambda^{2}$. The image of $W\left(\lambda_{0}\right)$ under that transform contains the domain

$$
\Omega_{0}(h):=\left[1-\frac{3}{4} h d\left(h^{-1}\right), 1+\frac{3}{4} h d\left(h^{-1}\right)\right]+i\left[0, \frac{3}{2} d\left(h^{-1}\right) h^{N^{\#} / 2+1+\varepsilon}\right]
$$

and there are no resonances $z$ of $P(h)$ in this domain. By Proposition $1, R_{\chi}(z)$ satisfies the exponential estimate (5) with $n^{\#}$ replaced by $N^{\#}$ in the smaller domain

$$
\Omega(h):=\left[1-\frac{1}{2} h d\left(h^{-1}\right), 1+\frac{1}{2} h d\left(h^{-1}\right)\right]+i\left[0, d\left(h^{-1}\right) h^{N^{\#} / 2+1+\varepsilon}\right],
$$

because the distance between $\Omega(h)$ and the closest resonance is at least $g(h)=$ $\frac{1}{2} d\left(h^{-1}\right) h^{N^{\#} / 2+1+\varepsilon}$ and $\ln (1 / g(h))=\ln 2+\ln \left(1 / d\left(h^{-1}\right)\right)+\left(N^{\#} / 2+1+\varepsilon\right) \ln (1 / h)$ $\leq C h^{-q}$. Set $5 w(h):=\frac{1}{2} h d\left(h^{-1}\right)$. Then we can apply Lemma 1 to get

$$
\begin{aligned}
& \left\|R_{\chi}(z)\right\| \leq \frac{C h^{3 N^{\#} / 2+1+2 \varepsilon}}{d\left(h^{-1}\right)}, \\
& \quad \text { for } z \in\left[1-\frac{1}{10} h d\left(h^{-1}\right), 1+\frac{1}{10} h d\left(h^{-1}\right)\right]+i\left[0, \frac{1}{10} d\left(h^{-1}\right) h^{3 N^{\#} / 2+1+2 \varepsilon}\right],
\end{aligned}
$$

for $h \ll 1$. Applying the inverse transform $\lambda=h^{-1} z^{1 / 2}=\lambda_{0} z^{1 / 2}$, we get the required estimate for $\lambda \in \tilde{\Omega}(h)$ and in particular for $\lambda=\lambda_{0}\left(1+\frac{i}{2}\left(\frac{1}{10} d\left(\lambda_{0}\right)\right.\right.$ $\left.\left.\lambda_{0}^{-3 n^{\#} / 2-1-2 \varepsilon}\left(1+O\left(\lambda_{0}^{-1}\right)\right)\right)\right)$. Replacing $2 \varepsilon$ by $\varepsilon$, we complete the proof of the proposition. 
Next proposition shows that although the resolvent may not be polynomially bounded near the real axis, integral of it over bounded intervals is.

Proposition 3. Assume (23). Then for $\mu>0$ large enough

$$
\begin{aligned}
& \int_{\mu}^{\mu+1}\left\|R_{\chi}(\lambda+i \alpha)\right\|_{\mathcal{D}^{M} \rightarrow \mathcal{H}} d \lambda \leq C \mu^{\frac{5 N^{\#}}{2}}-1+\varepsilon-2 M \\
& \qquad \text { for } 0 \leq \alpha \leq \frac{\min \{d(\lambda) ; \mu \leq \lambda \leq \mu+1\}}{22 \mu^{\frac{3 N \#}{2}+\varepsilon} .}
\end{aligned}
$$

Proof. Let $\mu$ be as above. We can assume that $\operatorname{dist}(\lambda, \mathcal{R}(P)) \leq 1$ for $\lambda \gg 1$, otherwise the estimate follows easily from Lemma 1. So, $d(\mu)=\operatorname{dist}(\mu, \mathcal{R}(P))$ for $\mu \gg 1$. By Proposition 2 and (19), for $\alpha$ as above,

$$
\left\|R_{\chi}(\lambda+i \alpha)\right\|_{\mathcal{D}^{M} \rightarrow \mathcal{H}} \leq \frac{C \lambda^{\frac{3 N \#}{2}-1+\varepsilon-2 M}}{d(\lambda)} \leq C \lambda^{\frac{3 N^{\#}}{2}-1+\varepsilon-2 M} \sum_{j} \frac{1}{\left|\lambda-\lambda_{j}\right|},
$$

where the summation is taken over all resonances satisfying $\lambda_{j} \in[\mu-1, \mu+2]+$ $i[0,2]$, if $\mu \leq \lambda \leq \mu+1$. According to (3), there are $O\left(\mu^{n^{\#}}\right)$ such resonances. We therefore get

$$
\begin{aligned}
& \int_{\mu}^{\mu+1}\left\|R_{\chi}(\lambda+i \alpha)\right\|_{\mathcal{D}^{M} \rightarrow \mathcal{H}} d \lambda \\
\leq & C \mu^{\frac{3 N \#}{2}-1+\varepsilon-2 M} \sum_{j} \int_{\mu}^{\mu+1} \frac{d \lambda}{\left|\lambda-\lambda_{j}\right|} \\
= & C \mu^{\frac{3 N \#}{2}-1+\varepsilon-2 M} \sum_{j} \int_{\mu}^{\mu+1} \frac{d \lambda}{\sqrt{\left(\lambda-\operatorname{Re} \lambda_{j}\right)^{2}+\left(\operatorname{Im} \lambda_{j}\right)^{2}}} \\
\leq & C \mu^{\frac{3 N \#}{2}-1+\varepsilon-2 M} \mu^{n^{\#}} \max _{j} \ln \frac{1}{\operatorname{Im} \lambda_{j}} \leq C \mu^{\frac{3 N^{\#}}{2}-1+\varepsilon+n^{\#}+q-2 M} .
\end{aligned}
$$

This proves the proposition.

We are ready now to prove an improved version of estimate (22) by lifting the lower part of $\Gamma_{l}$ above the real axis. More precisely, we replace the contour $\Gamma_{l}$ there by the boundary $\Gamma_{l}^{\prime}$ of the rectangle (compare with (16))

$$
\begin{aligned}
a_{l}-\frac{1}{2} a_{l}^{-k+1} & \leq \operatorname{Re} \lambda \leq b_{l}+\frac{1}{2} a_{l}^{-k+1}, \\
\frac{1}{23} d_{l} a_{l}^{-3 N^{\#} / 2-\varepsilon} & \leq \operatorname{Im} \lambda \leq \frac{1}{2} a_{l}^{-k-5 n^{\#} / 2-\varepsilon / 2+1},
\end{aligned}
$$

where $d_{l}=\min _{j}\left(\operatorname{Im} \lambda_{j}\right)$, with the minimum taken over all resonances $\lambda_{j}$ with real parts in $I_{l}=\left[a_{l}, b_{l}\right]$. Arguing as in (22) and using Proposition 3, we get for $2 M>5 N^{\#} / 2+1+\varepsilon$

$$
\left\|w_{l}(t, \cdot)\right\| \leq C e^{-t \alpha_{l}} \lambda^{-2}\left|\Gamma_{l}\right|\|g\|_{\mathcal{D}^{M}} \leq C e^{-t \alpha_{l}} \lambda^{-2}\left|I_{l}\right|\|g\|_{\mathcal{D}^{M}}
$$

where $\alpha_{l}:=\frac{1}{23} d_{l} a_{l}^{-3 N^{\#} / 2-\varepsilon}$ and $\lambda \in I_{l}$. 
This estimate implies the following.

Theorem 2. Under the assumptions of Theorem 1, assume also that $\operatorname{Im} \lambda_{j} \geq$ $S\left(\operatorname{Re} \lambda_{j}\right)$ with a decreasing positive function $S$, such that $-S^{\prime}(\lambda) / S(\lambda) \leq C \lambda^{q-1}$ for $\lambda>0$ large enough. Assume also that $K>7 n^{\#} / 2+q-1$. Then $\forall \varepsilon>0$, $\exists c>0$ such that we have the following estimate for $1 \ll A<B$

$$
\begin{aligned}
& \sum_{I_{l} \subset[A, B]}\left\|\sum_{\substack{\lambda_{j} \in \mathcal{R}(P) ; \operatorname{Re} \lambda_{j} \in I_{l} \\
\operatorname{Im} \lambda_{j}<\langle\lambda\rangle-K}} \chi \operatorname{Res}\left\{e^{i t \lambda} R(\lambda), \lambda_{j}\right\} \chi g\right\| \\
& \leq C \int_{A}^{B} \frac{e^{-t \tilde{S}(x)}}{x^{2}} d x\|g\|_{\mathcal{D}^{M}}, \quad \tilde{S}(x):=c|x|^{-3 N^{\#} / 2-\varepsilon} S(x)
\end{aligned}
$$

for any $g \in \mathcal{D}^{M}$ with $2 M>\max \left\{K+1,5 N^{\#} / 2+1+\varepsilon\right\}, N^{\#}=n^{\#}+q$.

Proof. The theorem follows directly from (25). Under the assumptions of the theorem, (23) is satisfied. Thus $d_{l}=\operatorname{Im} \lambda_{j_{0}} \geq S\left(\operatorname{Re} \lambda_{j_{0}}\right)$, where $\lambda_{j_{0}}$ is a resonance with real part in $\left[a_{l}, b_{l}\right]$. Our assumption on $S$ implies that $S(\lambda) \leq C S(\lambda+h)$ for $h=O\left(\lambda^{1-q}\right), \lambda \gg 1$. Tracing back the construction of $I_{l}$, we see that $\left|I_{l}\right|=$ $O\left(\lambda^{1-q}\right)$ for $K$ as in the theorem. This implies $d_{l} \geq c S(\lambda)$ for any $\lambda \in I_{l}$ with $c>0$ independent of $l$ and $\lambda$. Therefore, in (25) we have $\alpha_{l}>c S(\lambda) \lambda^{-3 N^{\#} / 2-\varepsilon}$, $\forall \lambda \in I_{l}$. This implies (26) easily.

\section{Rayleigh resonances}

Let $\Omega \subset \mathbf{R}^{n}$ be the complement of a strictly convex obstacle and consider the elasticity system with Neumann boundary conditions. The elasticity operator $\Delta_{e}$, acting on vector valued functions, has the form

$$
\Delta_{e} v=\mu_{0} \Delta v+\left(\lambda_{0}+\mu_{0}\right) \nabla(\nabla \cdot v),
$$

where $\lambda_{0}$ and $\mu_{0}$ are the Lamé constants satisfying $\mu_{0}>0, n \lambda_{0}+2 \mu_{0}>0$. We denote by $P$ the self-adjoint realization of $P$ with Neumann boundary conditions on the boundary $\Gamma=\partial \Omega$

$$
(B v)_{i}:=\left.\sum_{i=1}^{n} \sigma_{i j}(v) \nu_{j}\right|_{\Gamma}=0, \quad i=1, \ldots n,
$$

where $\sigma_{i j}(v):=\lambda_{0} \nabla \cdot v \delta_{i j}+\mu_{0}\left(\partial_{x_{j}} v_{i}+\partial_{x_{i}} v_{j}\right)$ is the stress tensor and $\nu$ is the outer normal to $\Gamma$.

It is known [SV1] that in this case there exist a sequence of resonances $\lambda_{j}$ with $0<\operatorname{Re} \lambda_{j}=O\left(\left|\lambda_{j}\right|^{-\infty}\right)$ and a symmetric sequence $-\bar{\lambda}_{j}$. This result is proven in [SV1] for $n=3$ but it also holds in any space dimension (see also [SjV]). If the boundary is analytic, the convergence is at an exponential rate [Vo]. There is also a logarithmic resonance free zone, i.e., there are no other resonances in $\Lambda:=\left\{\operatorname{Im} \lambda \leq C_{1} \ln \operatorname{Re} \lambda-C_{2}\right\}$ with some $C_{1}>0, C_{2}>0$. Moreover, there is an asymptotic formula for the counting function $N(r)=\{\lambda$-resonance, $\lambda \in \Lambda,|\lambda| \leq$ $r\}$ of the form $N(r)=C r^{n}+O\left(r^{n-1}\right)$, as $r \rightarrow \infty$ (see $\left.[\mathrm{SjV}]\right)$. Resonances with this density exist for arbitrary boundary as well [SV2], [S2] but then we may 
not have a resonance free zone. Existence of those resonances can be explained by the existence of Rayleigh surface waves propagating on the boundary with speed $C_{R}$ slower than the two sound speeds of the elasticity system. Those surface waves trap the energy near the boundary and in particular, there are singularities propagating on the boundary $[\mathrm{T}]$.

An application of Theorem 1 immediately yields a resonance expansion of the solution operator $U(t)$ for this system. This result also holds under the weaker assumptions on the geometry of the boundary considered in $[\mathrm{SjV}]$ that require a polynomial resonance free region for the Dirichlet problem (there are no surface waves for the Dirichlet problem) and an additional assumption on the Neumann operator. In the case of a strictly convex obstacle one can actually improve the estimate on the remainder. Denote by $\mathcal{N}(\lambda)$ the Neumann operator related to this system defined as follows

$$
\mathcal{N}(\lambda): H^{s}(\Gamma) \ni f \mapsto B v \in H^{s-1}(\Gamma),
$$

where $v$ is the $\lambda$-outgoing solution of the equation $\left(\Delta_{e}+\lambda^{2}\right) v=0$ in $\Omega$ satisfying $v=f$ on $\Gamma$ (see also [SV1]). In [SV1] it is shown that

$$
\left\|\mathcal{N}^{-1}(\lambda)\right\| \leq \frac{C}{\ln |\lambda|} \quad \text { for } \operatorname{Im} \lambda=a \ln |\operatorname{Re} \lambda|,|\operatorname{Re} \lambda|>2,
$$

with any fixed $a>0$ and $\|\cdot\|$ can be any $H^{s}$ norm, $s \geq 0$. Let $R_{D}(\lambda)$ be the outgoing Dirichlet resolvent and let $K_{D}(\lambda): f \rightarrow u$ be the outgoing solution operator of the homogeneous problem with Dirichlet data $f$ on $\Gamma$. Then

$$
R(\lambda)=R_{D}(\lambda)-K_{D}(\lambda) \mathcal{N}^{-1}(\lambda) B R_{D}(\lambda),
$$

where $R(\lambda)$ is the Neumann outgoing resolvent related to $P$ (see e.g. $[\mathrm{SjV}]$ ). Now we can use the fact that $R_{D}(\lambda)$ and $K_{D}(\lambda)$ are polynomially bounded in a logarithmic neighborhood of the real axis because the Dirichlet problem is non-trapping. This, together with (27), allows us to conclude that $R_{\chi}(\lambda)$ is polynomially bounded on $\operatorname{Im} \lambda=a \ln |\operatorname{Re} \lambda|$. Let $\Gamma$ be as above. Then the region above $\Gamma$ and below the curve $\operatorname{Im} \lambda=a \ln |\operatorname{Re} \lambda|,|\operatorname{Re} \lambda| \gg 1$ is free of resonances [SV1]. By the Phragmén-Lindelöf principle, $\left\|\mathcal{N}^{-1}(\lambda)\right\|$ is polynomially bounded there. This allows us to lift the contour of integration from $\Gamma$ to a line $\operatorname{Im} \lambda=$ const. $>0$ to obtain an exponential bound for the error term. As a result we get the following.

Theorem 3. Let $U(t)$ be associated with the Neumann problem in linear elasticity, assume that the obstacle $\mathcal{O}$ is strictly convex and let $s>(7 n / 2+1)$. Then for any $A>0$,

$$
\begin{aligned}
& \chi U(t) \chi g \\
& \quad=-i \sum_{l=1}^{\infty} \sum_{\substack{\lambda_{j} \in \mathcal{R}(P) ; \operatorname{Re} \lambda_{j} \in I_{l} \\
\operatorname{Im} \lambda_{j}<A}} \chi \operatorname{Res}\left\{e^{i t \lambda} R(\lambda), \lambda_{j}\right\} \chi g+E_{K}(t) g, \quad g \in H^{s},
\end{aligned}
$$


where the error term $E_{K}(t)$ satisfies $\left\|E_{K}(t)\right\|_{H^{s} \rightarrow L^{2}} \leq C e^{-(A-\varepsilon) t}, \varepsilon>0, n$ odd, and $\left\|E_{K}(t)\right\|_{H^{s} \rightarrow L^{2}} \leq C t^{-n+1}, n$ even. Here $I_{l}$ are as in Theorem 1 such that all resonances in $\operatorname{Im} \lambda<A$ have real parts in $\cup I_{l}$.

Theorem 3 admits the following interpretation: near the boundary, each smooth enough solution of the elastic wave equation with Neumann boundary conditions is a superposition of Rayleigh waves plus an exponentially decaying term.

4.1. The $3 \mathrm{D}$ case. In what follows we will restrict next ourselves to the $3 \mathrm{D}$ case where it is known [S2] that the resonances near the real axis are $O\left(|\lambda|^{-\infty}\right)$ perturbations of the eigenvalues of a self-adjoint classical $\Psi$ DO $P$ on the boundary with principal symbol $c_{R}|\xi|$. They are also the poles of the Neumann operator $\mathcal{N}(\lambda)$. Our main result here is (39) which gives a formula for the $w_{l}$ 's modulo an error term. We assume below that $\lambda \in \Lambda$.

As shown in [SV1], one can construct a parametrix for the Neumann operator $\mathcal{N}(\lambda)$. Here we are using pseudodifferential operators with large parameter $\lambda \in \Lambda$ and we will denote the corresponding class by $L^{m, k}$ (see e.g. [SV1], [SjV]). We have five microlocal regions related to $P$ because the elasticity operator has two wave speeds - a hyperbolic one, an elliptic one, two glancing ones and a mixed one which is hyperbolic with respect to one of the wave speeds and elliptic withe respect to the other one. The parametrix has a characteristic variety $\Sigma:=\left\{c_{R}|\xi|_{x}=1\right\}$ in the elliptic region and is elliptic or hypoelliptic in the other regions (see [SV1]) for more details). Moreover, near $\Sigma$ we have the following: if $\mathrm{WF}_{\lambda}(X)$ is near $\Sigma$, then for the parametrix $N_{e}$ in the elliptic region we have in block form (see [S2])

$$
V^{*}(\lambda) N_{e}(\lambda) V(\lambda) X(\lambda)=\left(\begin{array}{cc}
A-\lambda & 0 \\
0 & Q(\lambda)
\end{array}\right) X(\lambda)+R(\lambda) .
$$

Here $V(\lambda)$ is a classical $\Psi D O$ and $V(\lambda) \in \Psi^{1}$ uniformly in $\lambda$, invertible for large $\lambda$ uniformly in $\lambda, A=c_{R}\left(-\Delta_{\Gamma}\right)^{\frac{1}{2}} \bmod \Psi^{0}$ is self-adjoint independent of $\lambda$ and $Q(\lambda) \in \mathrm{L}^{1,1}$ is elliptic and self-adjoint for real $\lambda, R(\lambda)=O\left(|\lambda|^{-\infty}\right)$ is smoothing. For $\mathcal{N}(\lambda)$ we have

$$
\mathcal{N}(\lambda)=N(\lambda)+R(\lambda),
$$

where $R(\lambda)$ stands for (another) smoothing operator with norm $O\left(|\lambda|^{-\infty}\right)$ in each $H^{s}$ space. Here $N(\lambda)$ is the parametrix constructed using the parametrices in each region via a suitable partition of unity.

Proposition 4. There exists a function $0<S(\lambda)=O\left(|\lambda|^{-\infty}\right)$, such that

$$
\left\|\mathcal{N}^{-1}(\lambda)\right\| \leq \frac{C}{\operatorname{dist}(\lambda, \operatorname{spec} A)-S(\lambda)} \quad \text { for } \lambda \in \Lambda, \operatorname{dist}(\lambda, \operatorname{spec} A)>S(\lambda) .
$$

Sketch of the Proof. As in [SV1], we estimate $\mathcal{N} f$ from below in all microlocal regions. If $X$ is a $\lambda-\Psi D O$ with wave front set outside the characteristic variety 
$\Sigma:=\left\{c_{R}|\xi|=1\right\}$, then we have

$$
\|X f\| \leq C|\lambda|^{-2 / 3+\varepsilon}\|\mathcal{N} f\|+O\left(|\lambda|^{-\infty}\right)\|f\|, \quad \lambda \in \Lambda .
$$

Outside the glancing regions we have $O\left(|\lambda|^{-1}\right)$ in the first term. If $\mathrm{WF}_{\lambda}(X)$ is near $\Sigma$, then we can use (30) to get as in $[$ SV1, (5.5)-(5.7)]

$$
\operatorname{dist}(\lambda, \operatorname{spec} P)\|X f\| \leq C\|\mathcal{N} f\|+O\left(|\lambda|^{-\infty}\right)\|f\| .
$$

Therefore,

$$
\|X f\| \leq C \operatorname{dist}(\lambda, \operatorname{spec} P)^{-1}\left(\|\mathcal{N} f\|+O\left(|\lambda|^{-\infty}\right)\|f\|\right), \quad \lambda \in \Lambda .
$$

Here $X$ has a symbol supported near $\Sigma$ in the elliptic region. Combining (32) and (34), we get

$$
\|f\| \leq C \operatorname{dist}(\lambda, \operatorname{spec} P)^{-1}\left(\|\mathcal{N} f\|+O\left(|\lambda|^{-\infty}\right)\|f\|\right), \quad \lambda \in \Lambda .
$$

Here we used the fact that $\operatorname{dist}(\lambda, \operatorname{spec} P) \leq|\lambda|^{2 / 3-\varepsilon}, \lambda \in \Lambda,|\lambda| \gg 1$, because of the known asymptotics of $\operatorname{spec} P$. This implies the proposition.

Relations (30) and (31) imply that

$$
\mathcal{N}(\lambda) X(\lambda)=X_{1}(\lambda)\left(V^{*}\right)^{-1}(\lambda)\left(\begin{array}{cc}
A-\lambda & 0 \\
0 & Q(\lambda)
\end{array}\right) V^{-1}(\lambda)+R(\lambda)
$$

with $X$ and $R$ as in (30) and $X_{1}$ a zero order $\lambda$ - $\Psi$ DO such that this yields

$$
\begin{aligned}
& \mathcal{N}^{-1} X_{1}=X T^{-1}-\mathcal{N}^{-1} R T^{-1}, \text { where } \\
& T^{-1}(\lambda):=V(\lambda)\left(\begin{array}{cc}
(A-\lambda)^{-1} & 0 \\
0 & Q^{-1}(\lambda)
\end{array}\right) V^{*}(\lambda),
\end{aligned}
$$

By Proposition 4, if $\operatorname{dist}(\lambda, \operatorname{spec} A)>S(\lambda)+S_{1}(\lambda)$, then $\left\|\mathcal{N}^{-1}(\lambda)\right\| \leq C / S_{1}(\lambda)$. On the other hand, under the same assumption, $\left\|(A-\lambda)^{-1}\right\| \leq 1 /\left(S(\lambda)+S_{1}(\lambda)\right)$. Thus, for the remainder term above we get $\left\|\mathcal{N}^{-1} R T^{-1}\right\| \leq\|R\| / S_{1}^{2}$. Below we choose $S(\lambda)+S_{1}(\lambda)=C|\lambda|^{-k+1}, S(\lambda)=O\left(|\lambda|^{-\infty}\right)$, and this guarantees that $\|R\| / S_{1}^{2}=O\left(|\lambda|^{-\infty}\right)$ in this case.

Following similar arguments, we also get

$$
\mathcal{N}^{-1} \tilde{X}_{1}=O\left(|\lambda|^{-\infty}\right)
$$

if $\mathrm{WF}_{\lambda}\left(\tilde{X}_{1}\right) \cap \Sigma=\emptyset$ and $\lambda$ is separated from spec $A$ as above.

Let now $\lambda_{j}, j=1, \ldots, \infty$ be the resonances near the positive real axis. Since $\lambda_{j}$ are $O\left(\left|\lambda_{j}\right|^{-\infty}\right.$ ) perturbations of the eigenvalues $\mu_{j}$ of $A$ (see [S2]), the estimate on the remainder term in (36) and estimate (37) are valid if $\lambda \in \Lambda$ is at a distance at least $C|\lambda|^{-k}, k>0$ from the resonance set. Let $\Gamma_{l}$ be the boundary of the rectangle (compare with (15))

$$
a_{l}-\frac{1}{2} a_{l}^{-k+1} \leq \operatorname{Re} \lambda \leq b_{l}+\frac{1}{2} a_{l}^{-k+1}, \quad-\frac{1}{2} a_{l}^{-k+1} \leq \operatorname{Im} \lambda \leq \frac{1}{2} a_{l}^{-k+1} .
$$


Here $I_{l}=\left(a_{l}, b_{l}\right)$ are intervals as in section 3 and $k-1>n^{\#}=n=3$. By Proposition 4,

$$
\left\|\mathcal{N}^{-1}(\lambda)\right\| \leq C|\lambda|^{k-1} \quad \text { on each } \Gamma_{l} .
$$

Proposition 4 also implies that (38) is fulfilled in the gap between two consecutive $\Gamma_{l}$ 's, i.e., in $\left[b_{l}+a_{l}^{-k+1} / 2, a_{l+1}-a_{l+1}^{-k+1} / 2\right]+i[-1,1]$. This allows us to construct a contour $\Gamma$ as in section 3 and $\mathcal{N}^{-1}$ will satisfy (38) on $\Gamma$ and also on small vertical bars between two consecutive $\Gamma_{l}$ 's. By the symmetry, we have similar bounds near the resonances $-\bar{\lambda}_{j}$ close to the negative real axis. Let $B_{a}$ be a ball with radius $a \gg 1$ such that the obstacle is included in $B_{a}$ and denote $\Omega_{a}:=\Omega \cap B_{a}$. The following estimates

$$
\begin{aligned}
& R_{D}(\lambda)=O(|\lambda|): L^{2}\left(\Omega_{a}\right) \longrightarrow H^{2}\left(\Omega_{a}\right), \\
& K_{D}(\lambda)=O(|\lambda|): H^{1 / 2}(\Gamma) \longrightarrow H^{1}\left(\Omega_{a}\right), \quad \text { for }|\operatorname{Im} \lambda| \leq 1
\end{aligned}
$$

follow easily from the fact that the Dirichlet problem is non-trapping for the elasticity system and $R_{D}(\lambda)=O(1 /|\lambda|): L^{2}\left(\Omega_{a}\right) \longrightarrow L^{2}\left(\Omega_{a}\right)$. This allows us to conclude that on $\Gamma$ and on the verticals bars we have

$$
\left\|R_{\chi}(\lambda)\right\|_{\mathcal{D}^{M} \rightarrow \mathcal{H}}=O\left(|\lambda|^{-1-\epsilon}\right), \quad 2 M \geq k+\epsilon, \epsilon>0 .
$$

Since $k-1>n=3$, we get that in the three dimensional case, Proposition 4 holds with $s=5$ which is an improvement over the requirement on $s$.

We will use (36) and (37) to estimate

$$
w_{l}(t, x)=\frac{1}{2 \pi} \oint_{\Gamma_{l}} e^{i t \lambda} R_{\chi}(\lambda) g d \lambda
$$

(see also (21)). Since $A$ is self-adjoint, the algebraic multiplicity of each eigenvalue of $A$ is 1 (while the geometric multiplicity, i.e., the dimension of the associated eigenspace can be greater that 1 ). Using this, we find that

$$
\begin{aligned}
& w_{l}(t, x) \\
= & \sum_{\mu_{j} \in I_{l}} i e^{i t \mu_{j}} K_{D}\left(\mu_{j}\right) V\left(\mu_{j}\right) \operatorname{diag}\left(\Pi_{j}, 0\right) V^{*}\left(\mu_{j}\right) B R_{D}\left(\mu_{j}\right) g+R_{l}(t, x) g, \quad \forall l \gg 1,
\end{aligned}
$$

where $\Pi_{j}$ is the projection associated with the eigenvalue $\mu_{j}$ of $A$ and

$$
\left\|R_{l}(t, \cdot)\right\|_{\mathcal{D}^{M} \rightarrow \mathcal{H}}=e^{O\left(\lambda^{-\infty}\right) t} O\left(\lambda^{-\infty}\right), \quad \lambda \in I_{l}, \forall M>0 .
$$

In order to get (40), we used the fact that $k$ can be chosen to be any (large enough) number. Although this estimate is not uniform with respect to $t$, it shows that the remainder term $R_{l}(t, x)$ is uniformly $O\left(\lambda^{-\infty}\right)$ for $t$ in an interval of length larger that $C_{N} \lambda^{N}, \forall N>0$. If the boundary is analytic, then (40) is uniform for $0 \leq t \leq C e^{C \lambda}$ since the resonances in this case converge exponentially fast to the real axis [Vo]. It is unclear whether one can prove an estimate uniform in $t$ (this would probably require replacing the eigenvalues $\mu_{j}$ in the exponential term $e^{i t \mu_{j}}$ above by the resonances $\lambda_{j}$ ). Nevertheless, (39) gives the structure of $w_{l}(t, x)$ in this case. 


\section{Acknowledgments}

The author would like to thank Maciej Zworski for the numerous discussions on this subject and for his encouragement to write this paper.

\section{References}

[B1] N. Burq, Décroissance de lénergie locale de l'équation des ondes pour le problème extérieur et absence de résonance au voisinage du réel, Acta Math. 180 (1998), 129.

[B2] L Lower bounds for shape resonances widths of long range Schrödinger operators, preprint.

[BZ] N. Burq and M. Zworski, Resonance expansions in semi-classical propagation, preprint.

[CZ] T. Christiansen and M. Zworski, Resonance Wave Expansions: Two Hyperbolic Examples, Comm. Math. Phys. 212 (2000), 323-336.

[LP] P. D. Lax and R. Phillips, Scattering Theory, Pure and Applied Mathematics 26, New York, Academic Press, 1967.

[PV] G. Popov, G. Vodev, Georgi Resonances near the real axis for transparent obstacles, Comm. Math. Phys. 207(1999), 411-438.

[Sj] J. Sjöstrand, A trace formula and review of some estimates for resonances, Microlocal analysis and Spectral Theory (Lucca, 1996), 377-437, NATO Adv. Sci. Inst. Ser. C Math. Phys. Sci. 490, Kluwer Acad. Publ., Dordrecht, 1997.

[SjV] J. Sjöstrand and G. Vodev, Asymptotics of the number of Rayleigh resonances, Math. Ann. 309 (1997), 287-306.

[SjZ] J. Sjöstrand and M. Zworski, Complex scaling and the distribution of scattering poles, J. Amer. Math. Soc. 4 (1991), 729-769.

[S] P. Stefanov, Quasimodes and resonances: sharp lower bounds, Duke Math. J. 99 (1999), 75-92.

[S2] - Lower bound of the number of the Rayleigh resonances for arbitrary body, Indiana Univ. Math. J. 49 (2000), 405-426.

[SV1] P. Stefanov and G. Vodev, Distribution of resonances for the Neumann problem in linear elasticity outside a strictly convex body, Duke Math. J. 78 (1995), 677-714.

[SV2] _ Neumann resonances in linear elasticity for an arbitrary body, Comm. Math. Phys. 176 (1996), 645-659.

[TZ1] S.-H. Tang and M. Zworski, From quasimodes to resonances, Math. Res. Lett. 5 (1998), 261-272.

[TZ2] _ Resonance expansions of scattered waves, Comm. Pure Appl. Math. 53 (2000), 1305-1334.

[T] M. Taylor, Rayleigh waves in linear elasticity as a propagation of singularities phenomenon, Partial differential equations and geometry (Proc. Conf., Park City, Utah, 1977), Dekker, New York, 1979, pp. 273-291.

[Vo] G. Vodev, Existence of Rayleigh resonances exponentially close to the real axis, Ann. Inst. H. Poincar Phys. Thor. 67 (1997), 41-57.

[Va1] B. Vainberg, Asymptotic methods in equations of mathematical physics, Gordon \& Breach, New York, 1989.

[Va2] L Long time behavior of solutions to linear and nonlinear hyperbolic problems, Modern mathematical methods in diffraction theory and its applications in engineering (Freudenstadt, 1996), 234-250, Methoden Verfahren Math. Phys. 42, Lang, Frankfurt am Main, 1997.

[Z] M. Zworski, private communication, 1992.

Department of Mathematics, Purdue University, West Lafayette, IN 47907.

E-mail address: stefanov@math.purdue.edu 